\title{
A Comparative Study of Wireless Communication Protocols in a Computer Vision System for improving the Autonomy of the Visually Impaired
}

\author{
S. Tsantilas*, C. Spandonidis, F. Giannopoulos, N. Galiatsatos, D. Karageorgiou and C. Giordamlis \\ Prisma Electronics S.A., Dimokratias Av., 68132, Alexandroupoli, Greece
}

Received 1 August 2019; Accepted 28 December 2019

\begin{abstract}
The latest advancements in computer vision have enabled the development of new applications in numerous scientific and technological fields. The program "e-Vision" builds on these advancements with the aim to improve the autonomy of people with visual impairment by means of visual recognition and audio feedback. This is achieved by a set of sensors, properly embedded to a pair of glasses for capturing visual information and for transmitting auditory feedback messages. Appropriate software running on a mobile device (smartphone) is responsible for transmitting and receiving the audio and visual information to and from the sensors. This paper presents a comparative study of relevant wireless communication protocols that could be implemented for the communication of the sensors with the smart mobile device. The application demands certain criteria to be met, regarding data transmission rates, transmission range, and power consumption. The current study focuses on the comparison of the numerous existing wireless communication protocols. It is shown that Bluetooth and WiFi are the most advantageous in order to implement the audio and visual data transmission respectively.
\end{abstract}

Keywords: e-Vision, Computer Vision, Smart Glasses, Wireless Communication, Data Rate

\section{Introduction}

Globally it is estimated that approximately 217 million people have moderate to severe vision impairment and 36 million are blind [1]. Visually impaired people are constantly facing problems that inhibit them from performing even basic everyday tasks, most notably related to navigation, orientation, and communication.

$\mathrm{e}-\mathrm{V}$ ision builds on the latest impressive advancements of computer vision with the aim to improve the autonomy of people with visual impairment at both practical and emotional level. More specifically, its purpose is to develop an assistive system that will rely on visual information for recognition of the objects and faces surrounding the user. The system is capable of providing the user with real-time information relative to the corresponding image that is acquired at any time.

Computer vision has advanced to a point where its capabilities are comparable to those of human vision while keeping the demand for computing resources to a minimum. These technological advancements enable even small mobile devices with limited processing power such as smartphones to handle the required data processing. Moreover, society's ever-increasing trend of digitization produces a vast pool of available image data, which can be used to train machine learning algorithms.

Numerous systems have been developed over time for helping the visually impaired with their everyday tasks by employing a number of different sensors monitoring the person's surroundings. A large number of these systems are based on the premise that the sensors are embedded in a pair of glasses. The sensors can be cameras, microphones, distance

*E-mail address: rdprojects@prismael.com

ISSN: 1791-2377 @ 2020 School of Science, IHU. All rights reserved.

doi:10.25103/iestr.131.10 measuring etc. The suggested solutions attempt to tackle different problems. Many solutions have been proposed to tackle the problem of guidance in an urban environment. One approach is to provide the user with a pair of glasses or another wearable device, which carry one or more cameras to capture visual information about the immediate environment. The information is processed by appropriate algorithms and then certain audio messages regarding navigation are relayed to the user either via conventional earphones [2] - [3] or boneconducting headphones [4]. Other proposals focus on image or text recognition [5], [6], again conveying audio information to the user. There are solutions that aim to help the visually impaired in the task of finding specific objects [7] , [8] or even distinguish between different banknotes and coins [9], using computer vision algorithms. There are other proposals which combine the use of smart glasses with other devices such as intelligent walking canes [10] or specific tactile feedback devices [11]. Ultra-sonic sensors have also been implemented in similar assistive systems [12]. A different approached has been proposed in [13] where the information regarding the surroundings is captured by a set of time-of-flight sensors and then conveyed to the user as a binaural acoustic image representation.

While there have been significant advancements in systems that aim to assist in guidance and navigation, it is clear that the majority of these solutions are only capable of rudimentary obstacle recognition and avoidance. In the current literature, there is an apparent lack of proposals concerning the recognition of shapes or faces as semantic entities. The term "semantic entity" refers to the nature of an object, for example distinguishing between a chair and a table or the facial properties and identity of a person. Such an advancement in recognition technologies would enable people with visual impairments to perform everyday tasks such as shopping for groceries or, even more importantly have meaningful and emotional interactions. e-Vision proposes the 
development of a system that is based on computer vision software running on the user's smart mobile device. The visual information is fed to the device by a pair of glasses which carry an embedded camera. The processed information is passed to the user as audio cues through earphones (Fig. 1).

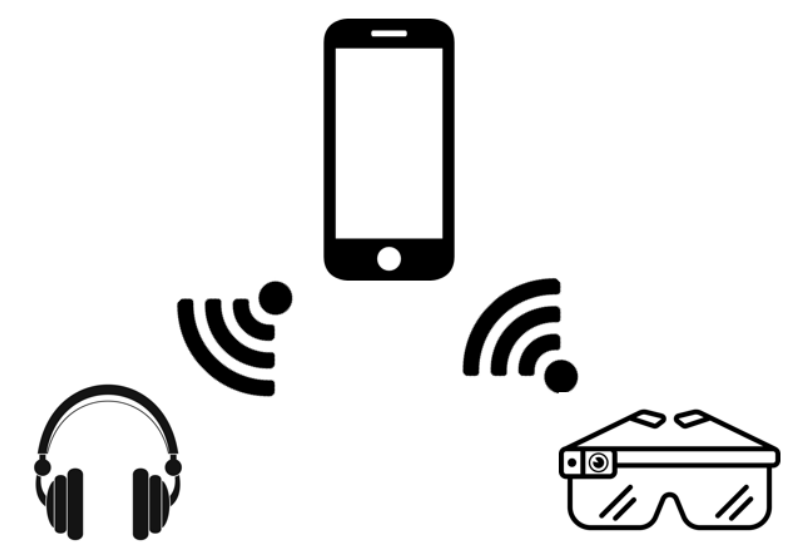

Fig. 1. Wireless communication between devices.

The communication between the sensors (camera, earphones) and the mobile device has to be handled through a wireless connection for the purpose of maintaining a userfriendly profile. In order to choose among the available wireless protocols, certain criteria have to be taken into consideration. These are: a) data transmission rate, b) range of communication, c) energy consumption, d) compatibility with available devices. Audio and video data rates will be studied in order to establish the minimum data rate which the wireless channel should be able to handle. Range of communication, while not being as critical, should also be considered. The system's energy consumption is an important metric since the whole apparatus must be powered by batteries and be able to operate for a reasonable amount of time. Lastly, the developed system's compatibility with commercially available devices must be taken into account.

In the following paragraphs, each of the aforementioned criteria is going to be examined for a number of widely used wireless communication protocols, while in the last paragraph a comparative study is presented for all communication protocols concerning these criteria. Before examining the wireless communication protocols in-depth, we present the data rate requirements that any wireless communication system must exhibit for transmitting and/or receiving audio and video. These two types of data are widely used in a vast number of applications and are quite often used in order to test wireless network characteristics.

Systems like e-Vision are based on providing audible information concerning a visually impaired person's environment. Thus, one of the most important characteristics of any such system is its ability to effectively transmit and receive audio.

\subsection{Data rate requirement}

The transmission rate of the audio and video data is the most important factor while considering the available wireless communication technologies. Audio and video signals have very different demands on bandwidth. As a result, they will be examined and analyzed independently.

\subsubsection{Audio}

In order to evaluate the requirements for the audio signal transmission, an initial assumption must be made concerning the desired quality of the signal. The audio channel will be used for the transmission of spoken information corresponding to the image that is being received. Hence, it is safe to assume that the wireless connection must be able to carry at least the bandwidth of the human voice. In the case of typical telephony, the sampling rate of the audio signal is 8 $\mathrm{kHz}$. Assuming the standard 16 bit per sample resolution the data rate is given by:

$$
8000 \frac{\text { samples }}{\text { sec }} \times 16 \frac{\text { bits }}{\text { sample }}=128 \text { Kbps }
$$

High Definition Voice standard defines a $16 \mathrm{kHz}$ sampling frequency. If HD Voice is to be used in order to achieve better audio quality the data rate is given by:

$$
16000 \frac{\text { samples }}{\text { sec }} \times 16 \frac{\text { bits }}{\text { sample }}=256 \mathrm{Kbps}
$$

\subsubsection{Video}

Three different video resolutions will be considered: $480 \mathrm{p}$, $720 \mathrm{p}$ and $1080 \mathrm{p}$. The standard refresh rate of 24 frames per second and 8-bit color depth is assumed. The bitrate calculation is given by the following [14]:

Pixels $_{H O R} \times$ Pixels $_{V E R} \times F P S \times($ Bit depth +2$) \times 3$

For the assumed $480 \mathrm{p}, 720 \mathrm{p}$ and $1080 \mathrm{p}$ resolutions the respective bitrates are $250 \mathrm{Mbps}, 670 \mathrm{Mbps}$, and $1500 \mathrm{Mbps}$.

\section{Wireless Communication Protocols}

\subsection{Bluetooth}

Bluetooth is a widely adopted communication protocol, found in most commercial mobile devices. It is also referred to as the IEEE 802.15.1 standard. The standard is maintained by the Bluetooth Special Interest Group (SIG). It is targeted towards implementing short-range, ad-hoc communication of devices such as computer peripherals, audio interfaces, mobile phones, etc. This type of communication is categorized as a wireless personal area network (WPAN). The smallest network defined by the Bluetooth standard is called a piconet. A piconet is created by a master device while several other devices can be connected as slaves on the same piconet. The piconet defines a star topology with up to 8 devices ( 7 slaves and 1 master), where all slaves communicate only with their master. Bluetooth operates in the $2.4 \mathrm{GHz}$ industrial, scientific and medical (ISM) band. In order to prevent interference from other networks operating in the same band, the transmission power does not exceed $1 \mathrm{~mW}$. Furthermore, a spread spectrum technique is employed in the form of frequency hopping. Hopping takes place among 79 distinct channels following a pseudorandom sequence [15].

\subsection{WiFi}

WiFi is the trade name of the IEEE 802.11 standard which describes wireless local area network (WLAN) protocols. It is the most common way for devices to connect wirelessly to local networks and to the internet. WiFi operates in the 2.4 $\mathrm{GHz}$ and $5 \mathrm{GHz}$ bands. In order to avoid interference with neighboring networks, the radio link is dynamically allocated into separate communication channels (13 channels in 
Europe, 11 in the USA and 14 in Japan) [15]. WiFi can operate either in ad hoc or infrastructure mode. In ad hoc mode there is no access point and all connections are peer-topeer. In infrastructure mode all devices connect to an access point (AP). This setup is commonly referred to as a basic service set (BSS).

\subsection{Zigbee}

Zigbee is a wireless communication protocol developed mainly for the purpose of creating a low power, multi-node, ad hoc networks such as personal area (PAN) and wireless sensor networks (WSN). It is defined by the IEEE 802.15.4 standard. Applications include home and industrial automation, collection of medical data, agricultural monitoring, etc. Zigbee operates in the $2.4 \mathrm{GHz}, 915 \mathrm{MHz}$ and $868 \mathrm{MHz}$ ISM bands. The devices in a Zigbee network are assigned specific roles. In every network, there must be only one main device (node) called the Zigbee Coordinator (ZC) which can provide a bridge to neighboring networks. The Zigbee Router (ZR) manages the traffic inside the network. The Zigbee End Device (ZED) is the last device in the Zigbee hierarchy. It stays in a low power state for most of the time and periodically wakes up to connect and exchange information with a ZR or ZC node [16].

\subsection{LoWPAN}

6LoWPAN stands for IPv6 over Low-Power Wireless Personal Area Network. The idea behind its inception is to enable even the smallest of devices to adopt the Internet Protocol (IP) [17]. It is designed for the purpose of developing IoT applications by enabling the transmission of IPv6 packets over the IEEE 802.15.4 standard. Deployments of 6LoWPAN comprise remote monitoring and control scenarios in-home or industrial environments and smart grid connectivity.

\subsection{IrDA}

The IrDA (Infrared Data Association) protocol describes the wireless communication which relies on the infrared part of the electromagnetic spectrum. The technology of infrared communication is ubiquitous, found on remote controllers, mobile devices, cameras, printers, and medical devices. Infrared communication characteristics are defined by its relatively small wavelength (850 nanometers to 900 nanometers) which impedes propagation through physical obstacles. As a result, the effective range is considerably smaller than that of competing implementations such as Bluetooth or $\mathrm{WiFi}$, while at the same time requiring line-ofsight communication with the devices being in alignment.

\section{Comparative study}

The comparison of the aforementioned protocols focuses on the criteria set in the introduction, i.e. the data rate, range, power consumption, and compatibility. A summary of the comparison is presented in Table 1 .

\subsection{Radio link characteristics}

Bluetooth has been revised several times throughout its existence. Bluetooth $2.0+$ EDR has a maximum theoretical data transmission rate of $3 \mathrm{Mbps}$, however, in practice, it can reach up to 2.1 Mbps. Revision 2.1 introduced easier connectivity and enhanced security. Bluetooth $3.0+\mathrm{HS}$ protocol. supports a maximum data rate of $24 \mathrm{Mbps}$ achievable by utilizing an 802.11 link for data transmission while reserving the Bluetooth link for connection management [18]. Bluetooth 4.0 introduced the low power specification known as Bluetooth Low Energy (BLE), targeting low data rate applications such as health and fitness monitoring. The realworld data rate of BLE is approximately 1 Mbps [19]. Bluetooth 5 is a relatively new revision developed mainly for the Internet of Things (IoT) applications. Its main feature is the increased range (up to $4 x$ the range of Bluetooth 4) that can be achieved by introducing a new physical layer which supports forward error correction (FEC). Error correction techniques introduce redundant information with direct impact on the maximum achievable data rate. In order to achieve the $4 \mathrm{x}$ range multiplier, the data rate is capped at 125 Kbps. On the other hand, the new physical layer supports double the data rate of previous versions reaching up to 2 Mbps with a $0.8 \mathrm{x}$ range multiplier [20].

As has been the case with Bluetooth, WiFi has had several revisions. $802.11 \mathrm{a}$ operates in the $5 \mathrm{GHz}$ band utilizing orthogonal frequency division multiplexing (OFDM) to improve the interference characteristics. $802.11 \mathrm{~b}, 802.11 \mathrm{~g}$, and $802.11 \mathrm{n}$ operate in the $2.4 \mathrm{GHz}$ band with each one improving on the data rate (up to $54 \mathrm{Mbps}$ ). The standard saw a significant increase in data rate with the introduction of 802.11ac which supports up to $1300 \mathrm{Mbps}$, operating in both the $2.4 \mathrm{GHz}$ and $5 \mathrm{GHz}$ bands [21]. WiFi Direct introduces a deviation from the standard WiFi philosophy enabling the communication between devices without the need for an access point. The result is an ad hoc network, similar to Bluetooth, with a data rate of up to $250 \mathrm{Mbps}$ [22].

Zigbee and 6LoWPAN are built upon the 802.15.4 standard which operates in 16 channels over the $2.4 \mathrm{GHz}$ band, in 10 channels over the $915 \mathrm{MHz}$ band and one channel over the $868 \mathrm{MHz}$ band. The maximum data rate of $250 \mathrm{Kbps}$ is achieved only at $2.4 \mathrm{GHz}$. At $915 \mathrm{MHz}$ the maximum data rate is $40 \mathrm{Kbps}$ and at $868 \mathrm{MHz}$ it is $20 \mathrm{Kbps}$ [23]. In infrared communication, the IrDA protocol supports $115.2 \mathrm{Kbps}$ data rate through the serial infrared (SIR) channel with version 1.0 physical layer and $4 \mathrm{Mbps}$ through the fast infrared channel (FIR) with version 1.1 physical layer [24] [25].

\subsection{Range and power consumption}

Bluetooth is mainly used in low power, short-range communications reaching up to 10 meters. Combined with its relatively high data rate capability it is ideal for mobile use cases, where the need for high-quality audio transmission arises. Low power variants (versions 4 and 5) achieve even more efficient operation by switching to a sleep state when not actively transmitting or receiving. Such behavior is well suited for low rate applications where small data is sparsely transmitted (for example when reading data from a sensor such as a heart rate monitor). $\mathrm{WiFi}$, on the other hand, is a much more power demanding

However, it can provide a range of 100 meters and a lot more capacity for high data rate exchanges. In fact, if we look at the normalized energy consumption $(\mathrm{mJ} / \mathrm{Mb}) \mathrm{WiFi}$ is about twice as efficient as Bluetooth [26]. While WiFi is more suited for WLANs, it is often found in ad hoc, mobile environments where high data rate communication is required. A typical example would be the real-time transmission of image data captured by a drone's camera to a mobile device (smartphone, tablet). 
S. Tsantilas, C. Spandonidis, F. Giannopoulos, N. Galiatsatos, D. Karageorgiou, C. Giordamlis/

Journal of Engineering Science and Technology Review 13 (1) (2020) 72 - 76

Table 1. Comparison of radio characteristics of the Bluetooth, WiFi, Zigbee, 6LoWPAN and IrDA protocols.

\begin{tabular}{|c|c|c|c|c|c|}
\hline & Bluetooth & WiFi & Zigbee & 6LoWPAN & IrDA \\
\hline IEEE $\mathrm{s}$ & 802.15 .1 & $802.11 \mathrm{a} / \mathrm{b} / \mathrm{g} / \mathrm{n} / \mathrm{ac}$ & 802.15 .4 & 802.15 .4 & \\
\hline $\begin{array}{l}\text { Frequency } \\
\text { band/Wavelength }\end{array}$ & $2.4 \mathrm{GHz}$ & $2.4 / 5 \mathrm{GHz}$ & $\begin{array}{l}2.4 \mathrm{GHz} \\
868 / 915 \mathrm{MHz}\end{array}$ & $\begin{array}{l}2.4 \mathrm{GHz} \\
868 / 915 \mathrm{MHz}\end{array}$ & $\begin{array}{l}850 \mathrm{~nm} \text { to } \\
900 \mathrm{~nm}\end{array}$ \\
\hline Max data rate & $\begin{array}{l}24 \text { Mbps (ver. } \\
\text { 3.0), } \\
2 \text { Mbps (BLE) }\end{array}$ & $\begin{array}{l}54 \text { Mbps }(802.11 \mathrm{n}) \\
1300 \mathrm{Mbps}(802.11 \mathrm{ac})\end{array}$ & $125 \mathrm{Kbps}$ & $125 \mathrm{Kbps}$ & $\begin{array}{l}4 \quad \text { Mbps } \\
\text { (FIR) }\end{array}$ \\
\hline Channel modulation & GFSK & $\begin{array}{l}\text { BPSK, QPSK } \\
\text { COFDM, CCK, M- } \\
\text { QAM }\end{array}$ & $\begin{array}{l}\text { O-QPSK }(2.4 \\
\text { GHz), } \\
\text { BPSK } \quad(915 / 868 \\
\text { MHz })\end{array}$ & $\begin{array}{l}\text { O-QPSK }(2.4 \\
\text { GHz }), \\
\text { BPSK } \quad(915 / 868 \\
\text { MHz })\end{array}$ & $\begin{array}{l}\text { RZI } \\
\text { PPM }\end{array}$ \\
\hline Data protection & $\begin{array}{l}\text { 24-bit CRC and } \\
\text { FEC (BLE) }\end{array}$ & 32-bit CRC and FEC & $\begin{array}{l}\text { 16-bit CRC and } \\
\text { FEC }\end{array}$ & $\begin{array}{l}\text { 16-bit CRC and } \\
\text { FEC }\end{array}$ & $\begin{array}{l}\text { 16-bit } \\
\text { CRC }\end{array}$ \\
\hline
\end{tabular}

Acronyms: GFSK (Gaussian frequency shift keying), BPSK (binary phase SK), QPSK (quadrature PSK), O-QPSK (offset QPSK), COFDM (coded orthogonal frequency division multiplexing), CCK (complimentary code keying), M-QAM (M-ary quadrature amplitude modulation), RZI (return to zero, inverted), PPM (pulse position modulation), CRC (cyclic redundancy check), FEC (forward error correction).

Zigbee and 6LoWPAN offer a typical range of 100 meters which can be extended by adding more nodes to the network. The nodes' absolute value of power consumption is rather high (comparable to that of WiFi). However, the typical applications where these protocols are deployed do not require always-on operation (for example controlling power outlets in a smart home application) thus achieving very low total consumption in the network [27] [28]. Obviously, such a solution would not be suitable for continuous transmission of data, even less so if there is the need for high data rates.

Infrared communication is very limited in range (about one meter) due to the nature of wave propagation in such small wavelengths. In addition to high free space propagation attenuation, there is also the requirement for line of sight communication between devices making it unsuitable for most use cases.

Table 2 summarizes the typical range and power consumption values of the wireless protocols.

Table 2. Comparison of range and power consumption of the Bluetooth, WiFi, Zigbee, 6LoWPAN and IrDA protocols.

\begin{tabular}{|c|c|c|c|c|c|}
\hline & Bluetooth & WiFi & Zigbee & 6LoWPAN & IrDA \\
\hline Nominal range & $10 \mathrm{~m}$ & $100 \mathrm{~m}$ & $100 \mathrm{~m}$ & $100 \mathrm{~m}$ & $1 \mathrm{~m}$ \\
\hline Nominal Tx power & -20 to $10 \mathrm{dBm}$ & $\begin{array}{l}15 \text { to } 20 \\
\mathrm{dBm}\end{array}$ & -25 to $0 \mathrm{dBm}$ & -25 to $0 \mathrm{dBm}$ & $\begin{array}{l}\text { approx. } \\
\text { mW }\end{array}$ \\
\hline $\begin{array}{l}\text { Typical power } \\
\text { consumption }\end{array}$ & $\begin{array}{l}\text { Less than } 10 \mathrm{~mW} \\
\text { (BLE) }\end{array}$ & $250 \mathrm{~mW}$ & $250 \mathrm{~mW}$ & $250 \mathrm{~mW}$ & $10 \mathrm{~mW}$ \\
\hline
\end{tabular}

\subsection{Compatibility with existing technology}

Nowadays there are a plethora of commercial devices that support any of the aforementioned protocols. One could safely assume that any modern mobile smart device (smartphone, tablet) natively provides Bluetooth and WiFi connectivity. There is already a vast selection of wireless headsets, speakers and cameras that offer direct connectivity with mobile devices for real-time data streaming, be it audio or video. On the other hand, 802.15.4 technologies like Zigbee and 6LoWPAN aren't usually supported directly, which implies the need for a dedicated gateway in order to provide connectivity with common commercial electronic devices. This introduces a generally unwanted complexity when designing a system that requires end-users to connect with their own smart devices.

\section{Conclusion}

e-Vision is a novel system that aims to improve the autonomy of people with visual impairment. The system comprises a set of sensors embedded in a pair of glasses that capture visual information and provide audio feedback to the user. The information must be handled and processed by the user's mobile smart device. One of the most important aspects of this novel system is the communication between the end user's glasses and his/her mobile device. Therefore, a very important first step towards the development of the system is the choice regarding the wireless communication protocol that is going to be employed. The comparative study presented in the present paper shows that among the competing wireless protocols there are two that completely fit the requirements of the application. In the case of the audio transmission, Bluetooth is the most appropriate protocol since it combines adequate data rates with low power consumption. As far as video transmission is concerned the most fitting protocol was found to be WiFi as it can handle the demanding task of transmitting high data rate signals. In addition, both Bluetooth and WiFi are natively supported by all contemporary smart devices, an attribute that is extremely convenient as it allows for direct connectivity without the use of a separate gateway device which would increase the complexity and power requirements of the system.

\section{Acknowledgments}

This research has been co-financed by the European Regional Development Fund of the European Union and Greek national funds through the Operational Program "Competitiveness, Entrepreneurship and Innovation, under the call RESEARCH CREATE INNOVATE (project code T1EAK-02454, titled "eVision").

This is an Open Access article distributed under the terms of the Creative Commons Attribution License 


\section{References}

[1] World Health Organization, "Blindness and vision impairment," 11 October 2018. [Online]. Available: https://www.who.int/en/news-room/fact-

sheets/detail/blindness-and-visual-impairment.

[2] A. Landa-Hernández and E. Bayro-Corrochano, "Cognitive guidance system for the blind," in World Automation Congress, 2012.

[3] W. M. Elmannai and K. M. Elleithy, "A novel obstacle avoidance system for guiding the visually impaired through the use of fuzzy control logic," in CCNC 2018 - 2018 15th IEEE Annual Consumer Communications and Networking Conference, Las Vegas, NV, USA, 2018.

[4] F. Lan, G. Zhai and W. Lin, "Lightweight smart glass system with audio aid for visually impaired people," in TENCON 2015 - 2015 IEEE Region 10 Conference, Macao, 2015.

[5] E. A. Hassan and T. B. Tang, "Smart Glasses for the Visually Impaired People," in Computers Helping People with Special Needs: 15th International Conference, ICCHP 2016, Linz, 2016.

[6] A. G. Sareeka, K. Kirthika, M. R. Gowthame and V. Sucharitha "pseudoEye Mobility assistance for visually impaired using image recognition," in 2018 2nd International Conference on Inventive Systems and Control (ICISC), Coimbatore, 2018.

[7] J. P. Bigham, C. Jayant, A. Miller, B. White and T. Yeh, "VizWiz::LocateIt - enabling blind people to locate objects in their environment," in 2010 IEEE Computer Society Conference on Computer Vision and Pattern Recognition - Workshops, San Francisco, CA, USA, 2010.

[8] B. Schauerte, M. Martinez, A. Constantinescu and R. Stiefelhagen, "An Assistive Vision System for the Blind That Helps Find Lost Things," in Computers Helping People with Special Needs. ICCHP 2012. Lecture Notes in Computer Science, vol 7383, Springer, Berlin, Heidelberg, 2012, pp. 566572 .

[9] A. Sirikham, W. Chiracharit and K. Chamnongthai, "Banknote and coin speaker device for blind people," in International Conference on Advanced Communication Technology, ICACT Phoenix Park, South Korea, 2009.

[10] L. Chen, J. Su, M. Chen, W. Chang, C. Yang and C. Sie, "An Implementation of an Intelligent Assistance System for Visually Impaired/Blind People," in 2019 IEEE International Conference on Consumer Electronics (ICCE), 2019.

[11] R. Velazquez, E. E. Pissaloux, J. C. Guinot and F. Maingreaud, "Walking Using Touch: Design and Preliminary Prototype of a Non-Invasive ETA for the Visually Impaired," in Engineering in Medicine and Biology Society (EMBC), Annual International Conference of the IEEE, Shanghai, 2005.

[12] A. Suresh, D. Laha, D. Gaba and S. Bhambri, "Robot Intelligence Technology and Applications," in Robot Intelligence Technology and Applications, vol. 5, 2019, pp. 99112 .

[13] L. Dunai, B. D. Garcia, I. Lengua and G. Peris-Fajarnes, "3D CMOS sensor based acoustic object detection and navigation system for blind people," in IECON Proceedings (Industrial Electronics Conference), Montreal, 2012.

[14] P. H. Putman, Guide to Wireless AV Connectivity, Kramer Electronics USA, 2014.

[15] S. Sattel, June 2019. [Online]. Available: https://www.autodesk.com/products/eagle/blog/wifi-vsbluetooth-wireless-electronics-basics/.
[16] "Zigbee End Device," 2010. [Online]. Available: https://www.sciencedirect.com/topics/computerscience/zigbee-end-device.

[17] Z. Shelby and C. Bormann, "6LoWPAN: The wireless embedded Internet - Part 1: Why 6LoWPAN?," 2011. [Online]. Available: https://www.eetimes.com/document.asp?doc_id=1278794.

[18] Bluetooth SIG, "Specification Documents," June 2019 [Online]. Available: http://www.bluetooth.org/docman/handlers/DownloadDoc.ash $\mathrm{x}$ ?doc_id=40560\&ei=25GiT8L3CuTa0QGnmqDVDA\&usg=A FQjCNGXY5pm4Tkju1KGs4dYRJLtd03FEg.

[19] M. Afaneh, "Bluetooth 5 speed: How to achieve maximum throughput for your BLE application," June 2019. [Online]. Available: https://www.novelbits.io/bluetooth-5-speedmaximum-throughput/.

[20] M. Woolley and S. Schmidt, "Bluetooth 5 Go Faster. Go Further," Bluetooth SIG, p. 25, 2016.

[21] IEEE, "New IEEE 802.11ac Specification Driven by Evolving Market Need for Higher, Multi-User Throughput in Wireless LANs," 2008. [Online].

[22] "Wi-Fi Alliance," 2019. [Online]. Available: https://www.wifi.org/discover-wi-fi/wi-fi-direct.

[23] R. Tabish, A. Ben Mnaouer, F. Touati and A. M. Ghaleb, "A comparative analysis of BLE and 6LoWPAN for U-HealthCare applications," in IEEE GCC Conference and exhibition, Doha, Qatar, 2013.

[24] P. Barker, A. C. Boucouvalas and V. Vitsas, "Performance modeling of the IrDA protocol for infrared wireless communications," International Journal of Communication Systems, vol. 36, no. 12, pp. 113-117, 2000.

[25] I. Millar, M. Beale, B. J. Donoghue, K. W. Lindstrom and S. Williams, "The IrDA standards for high-speed infrared communications," Hewlett-Packard Company, 1998.

[26] J.-S. Lee, Y.-W. Su and C.-C. Shen, "A Comparative Study of Wireless Protocols: Bluetooth, UWB, ZigBee, and Wi-Fi," in The 33rd Annual Conference of the IEEE Industrial Electronics Society (IECON), Taipei, Taiwan, 2007.

[27] J. Han, H. Lee and K.-R. Park, "Remote-Controllable and Energy-Saving Room Architecture based on ZigBee Communication," IEEE Transactions on Consumer Electronics, vol. 55, no. 1, 2009.

[28] J. Han, C.-S. Choi, W.-K. Park, I. Lee and S.-H. Kim, "Smart Home Energy Management System Including Renewable Energy Based on ZigBee and PLC," IEEE Transactions on Consumer Electronics, vol. 60, no. 2, 2014.

[29] L. Sang-Woong, K. Seonghoon and L. Seong-Whan, "A walking guidance system for the visually impaired," International Journal of Pattern Recognition and Artificial Intelligence, vol. 22, pp. 1171-1186, 2008.

[30] L. Everding, L. Walger, V. S. Ghaderi and J. Conradt, "A mobility device for the blind with improved vertical resolution using dynamic vision sensors.," in IEEE 18th International Conference one-Health Networking, Applications and Services (Healthcom), Munich, 2016.

[31] J. Bai, S. Lian, Z. Liu, K. Wang and D. Liu, "Smart guiding glasses for visually impaired people in indoor environment," IEEE Transactions on Consumer Electronics, vol. 63, pp. 258266, 2017. 